\title{
Glutathione (GSH) Production as Protective Adaptation Against Light Regime Radiation of Symbiodinium Natural Population
}

\author{
Moh. Muhaemin ${ }^{1}$, Dedi Soedharma ${ }^{2}$, Hawis Madduppa ${ }^{2}$ and Neviaty P. Zamani2*
}

\author{
Marine Science Department, University of Lampung \\ Jl. Sumantri Brojonegoro No. 01 Gedong Meneng-Bandar Lampung, Indonesia 35145 \\ ${ }^{2}$ Marine Science Department, Bogor Agricultural University \\ Jalan Lingkar Akademi, Kampus IPB Dramaga Bogor, Indonesia 16680 \\ Email: jurnalzamani@gmail.com
}

\begin{abstract}
Glutathione (GSH), as a wide range of low molecular weight, which found in marine microalgae and event bacteria, are essential to prevent photooxidation and productivity loss from these Radical Oxigen Species (ROS). Symbiodinium, endo-symbiont of corals, were exposed with different UV radiation combined with irradiance treatments to explore biomass specific initial response. Intracellular glutahione was observed as potential adaptive response of Symbiodinium population under environmental specific stress. The result showed that GSH production increased significantly with increasing irradiance and/or UV levels. GSH concentration was fluctuated among populations exposed by different irradiance treatments, but not effected by UV and irradiance exposure. GSH production as a response of UV exposure was higher than irradiance treatments. Both these high correlative fluctuation of intracellular GSH production and the presence of both treatments indicated protective specific adaptation of Symbiodinium under specific environmental stress, respectively.
\end{abstract}

Keywords: zooxanthellae, irradiance, glutathione (GSH), corals, Fungia

\section{Introduction}

Light is a key factor for the physiology of
corals that contain symbiotic phototropic
microorganisms (Symbiodinium). As such appropriate lightning is essential for growing and maintaining zooxanthellae corals in captivity. Symbiodinium cuture in relation to coral growth is the effect of rapid light fluctuations; short fluctuations in light intensity having an either stimulating or inhabiting effect on photosynthesis (Osinga et al., 2008). Photosynthetically Active Radiation (PAR) intensity, with wavelength in the range of $400-700 \mathrm{~nm}$, show a relationship with Symbiodinium photosynthesis (Krueger and Gates, 2012). A possitive correlation between PAR intensity and photosynthetic rate described while a plateau reached (the maximum photosynthetic capacity has been reached) and at the high PAR intensities, the photosynthesis rate decreased due to photoinhibition. These result imply that for efficient coral growth, a combination of high PAR intensity and regular feeding are needed. Based on the higher symbiotic mutualism relationship between coral and their zooxanthellae endosymbiont, most of coral food supplied by zooxanthellae. Coral regular feeding activity will increasing by the decreasing of zooxanthellae food supplay. Moya et al. (2006) showed that light is also known to enhance the calcification process in corals.
Light may effect production on the intracellular concentration of initial intracellular biochemical compound of marine microalgae (Foyer and Noctor, 2000). Researchers showed the potential role of thiols as ligands in seawater by measuring GSH and other thiols in marine microalgae field samplings (Tang et al., 2000; AlFarawati and van den Berg 2001; Muhaemin, 2008). The dynamic acclimation model of phytoplankton described the correlation of light, nutrient, and temperature (as independent factors) with the growth and intracellular biochemical compound (Tang et al., 2000). The high positive correlation of light dinamic and intracellular GSH concentration in marine microalgae. Dupont et al. (2004) and Muhaemin (2008) indicated that the intracellular GSH concentration in marine microalgae tend to increased in higher sunlight intensity

The tripeptide glutathione (GSH) is the main low molecular weight thiol in microalgae tissues. Due to the particular properties of the molecule, it plays multiple roles in cellular metabolism. It is a central compound in sulphur metabolism and is considered the main transport form of reduced sulphur (Tang et al., 2000). It links the sulphur reduction pathways to the protein synthesis and functions as a buffer for reduced sulphur. GSH also plays an important role in the scavenging of toxic reactive oxygen species (ROS). The activation of dioxygen is an important 
aspect of the cell metabolism. Oxidative attack initiates lipid peroxidation, protein oxidation, DNA damages, and pigment bleaching (Elstner and Oswald in Muhaemin, 2011).

Symbiodinium, a group of simbiotic microalgae in polyp of coral reef, may has ability to adapt with photon flux densities and UV in shalow coral reef system as indicated in coral growth model (Somathilake and Wedagedera, 2012). The adaptation mechanisms may involved photolyasemediated photo reactivation that repairs UV damaged DNA, UV screening along membrane, and turn over of repair cycle (Muhaemin 2011). Therefore, the photoadaptation of Symbiodinium to UV is considerable interest. In this research we analize the potential use of GSH as specific adaptive respon of Symbiodinium in the occurence combination of UV and irradiance.

\section{Materials and Methods}

The specimens of Fungia sp were collected from Pahawang Island waters, Lampung and transported to Aquaculture Research Laboratory, University of Lampung. The experimental acclimation were conducted at $26 \pm 1{ }^{\circ} \mathrm{C}$. The specimens were cultured in semi outdoor running seawater tank and fully exposed with ambient natural solar radiation 12:12 Light:Dark photoperiod ratio. The maximum photosynthetic photon flux density (PPFD) exposed to the specimens was 700-900 nm (2000 \pm 50 $\left.\mu \mathrm{mol} . \mathrm{m}^{-2} . \mathrm{s}^{-1}\right)$. The four treatments were tested with five replicates on each treatments. The treatments were low radiance with UV (LUV, 240-250 $\mu \mathrm{mol} . \mathrm{m}^{-}$ ${ }^{2} . \mathrm{s}^{-1}$ ), low radiance without UV (LWU, 70-80 $\mu \mathrm{mol} . \mathrm{m}^{-}$ $\left.2 . \mathrm{s}^{-1}\right)$, high radiance with UV (HUV, 290-300 $\mu \mathrm{mol} . \mathrm{m}^{-}$ $2 . \mathrm{s}^{-1}$ ), and high radiance without UV (HWU, 120-130 $\left.\mu \mathrm{mol} . \mathrm{m}^{-2} . \mathrm{s}^{-1}\right)$.

Isolated Symbiodinium from Fungia $s p$ were homogenized in tissue homogenizer by using Moore's calcium free artificial seawater with $\pm 0,03$ $\%(\mathrm{w} / \mathrm{v})$ sodium dodecyl sulfate as described in Krueger and Gates (2012). In order to minimize the contamination with host tissue and various unexpected microalgae, a half concentrated media of solid agar plates were tested with and without active aeration (Krueger and Gates, 2012) The homogenated samples were filtered through a Whatman GF/F filters (35 $\mu \mathrm{m}$ ) to remove clumps, washed, and centrifuged for 15 min with $110 \mathrm{mM}$ phosphate buffer $\mathrm{pH}$ 7.0. The culture media composition and preparation were based on formula given in Krueger and Gates (2012). The various media was added with respect to nitrogen source $\left(\mathrm{NO}_{3}{ }^{-} / \mathrm{NH}_{4}{ }^{-}\right)$, chelating agents and the combination of trace elements and organic supplements. Silica and Tris-base were omiteted from all media to minimize biocontamination by diatom. The Symbiodinium biomass were calculated by using Naubauer Counter Haemocytometer.

The particulate thiols were analized by preparing three $50 \mathrm{ml}$ and three $5 \mathrm{ml}$ aliquots of culture were gently filtered onto a Whatman GF/F filters at each sampling. Then sample filters were heated to $70{ }^{\circ} \mathrm{C}$ in $10 \mathrm{mmol} . \mathrm{I}^{-1}$ methanosulfonic acid (MSA) for 2 min and homogenized on ice (Dupont et al., 2004). The MSA extract was retained for derivatization with the fluorescence tag monobromobimane (Ahner et al., 2002).

Derivatized homogenate was taken then analyzed on a Beckman HPLC equipped with reversed-phased C-16 amide column and a $100 \mu \mathrm{l}$ injection loop. Compounds were quantified post column using fluorescence detection (excitation 310-410 nm; emission 474-650 nm). The elution gradient and buffers used are described elsewhere (Method C; Dupont et al., 2004). Standard curves for peak area calibration and to verify elution times was made by using stock solutions of cysteine and glutathione. For both, the detection limit is \pm 210 femtomol per injection (Muhaemin, 2008).

The biomass of Symbiodinium and GSH concentration were evaluated by t-test at the $5 \%$ significant level to identify the significance different treatment effect occured. The output consisted of a mean and standard deviation for each measurement within the population tested.

\section{Results and Discussion}

\section{Biomass of Symbiodinium}

The Symbiodinium biomass was inversely related to irradiance and the present of UV (Figure 1). The effect of irradiance were significant, and UV has no significant effect on Symbiodinium biomass fluctuation during research. The mean of Symbiodinium biomass exposed with UV treatments were always lower than without UV treatments, respectively. The different relative effect of irradiance and UV indicated the variation of Symbiodinium responses to both treatments. The present of UV and irradiance have a synergestic effect on Symbiodinium biomass. Althought, the significant effect of UV on Symbiodinium biomass was higher in high irradiance exposure than in the low irradiance exposure.

The irradiance in tolerable levels may induce the photosynthetic process. Tiltyanov et al. (2001) showed that the dynamic fluctuation of 


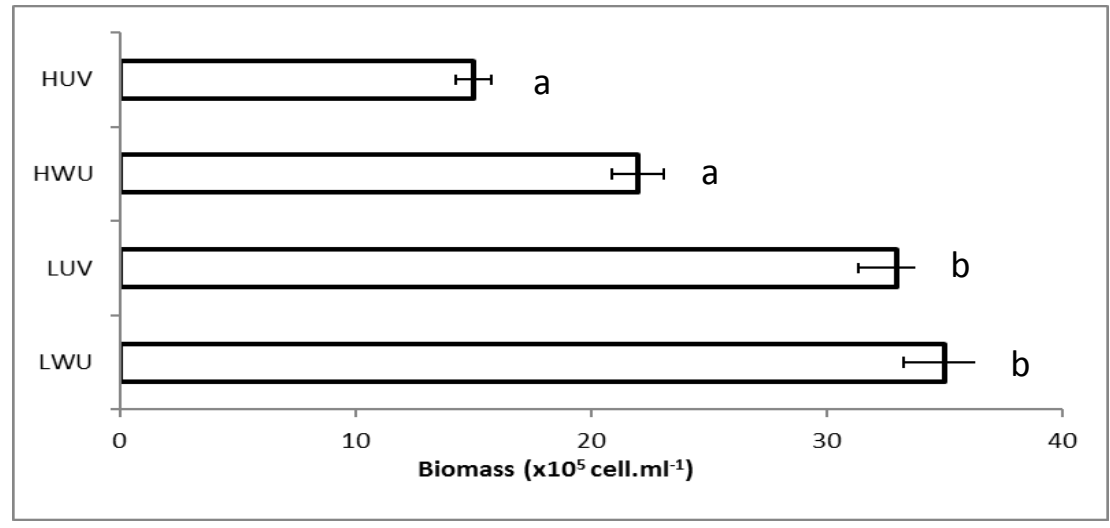

Figure 1. The average of Symbiodinium biomass ( $\times 10^{6}$ cell. $\left.\mathrm{ml}^{-1}\right)$ after 10 days of exposure. Different small text indicate the significant t-test result $(\alpha=0,05)$. Treatments: Low radiance with UV (LUV), low radiance without UV (LWU), high radiance with UV (HUV), and high radiance without UV (HWU)

zooxanthellae biomass as a result of photoacclimation process. The acclimation to light as a function of irradiance may involved the light harvesting dynamic capacity by increasing photosynthetic pigment concentration in zooxanthellae and regulated by changes in rates of division and degradation of symbiotic cells. The UV exposure regulated the slight degradation of biomass and chlorophyll concentation (Muhaemin, 2011). In addition, UV radition insensitivity may a result from other protection mechanism and cannot be solely correlated with the difference of UV capacity.

The abundance and diversity of Symbiodinium as a respond of environmental condition probably related with adaptive radiations mechanism driven by ecological specialization of the host (Finney et al., 2010), specific adaptation to micro habitat elevation (Perez et al., 2001), xanthophyll cycle as photoprotective agent (Latowski et al., 2012), and tolerate levels of zooxanthellae (Merwe et al., 2014). Osinga et al. (2008) showed that heterotropic feeding increases the photosyntetic capacity of Symbiodinium and corals tend to providing additional food have higher photosynthetic rates at higher PAR exposure than corals in starving level.

\section{GSH Concentration in Symbiodinium Cells}

Figure 2 described information about the shortterm adaptation to irradiance and UV stress condition within $10 \mathrm{~d}$ of culture. The combination treatment of high radiance with UV exposure has highest intracellular GSH concentration in Symbiodinimum biomass. The intracellular GSH produced by Symbiodinium biomass tend to vary related with the initial respond of both irradiance exposure (high and low irradiance). Treatment of UV at higher irradiance exposure tend to triggered higher production of intracellular GSH than lower irradiance exposure. Those showed that intracellular GSH production was higher in the combination with high irrradiance exposure. Symbiodinium biomass which growing at high elevations (of both treatments) often have higher constitutive intracellular GSH concentrations. Although the relative depletion effect of both treatments considerable has the same value. The dynamic of intracellular GSH concentration of Symbiodinium biomass may therefore be expected to play important roles for the cell with regard to the metabolic functions (Balarinova et al., 2014). The phenomena may correlated also with the lightdependence (photorespiration) of GSH accumulation in Symbiodinium cells. Photorespiration may therefore be important in supplying glycine when GSH synthesis is up-regulated by various stress conditions (Pinto et al., 2003).

Since ROS produced even under normal metabolic conditions, Symbiodinium may have developed effective defence systems to survive. Figure 2 showed that Symbiodinium exposed with high radiance of light and UV produced higher concentration of GSH in cells. In order to avoid or to minimize cellular destruction, specific mechanism must be taken by producing GSH. The GSH molecule has strong antioxidative properties (Muhaemin, 2008). It may react directly with ROS or with oxidized substrates reducing them and the antioxidative properties GSH may stabilize membrane structures (Pinto et al., 2003). Light intensity and UV radiation transmit more energy per quantum and are potentially dangerous to Symbiodinium. UV absorption may origenated to the direct production of ROS at the cell surfaces (e. g. directly at the plasmalemma) and or occur by increasing the ROS production in chloroplasts like most other stress is impacts as assumed. With increasing stress, cell metabolism severely disrupted. When membranes and cell compartments are destroyed, the chain 


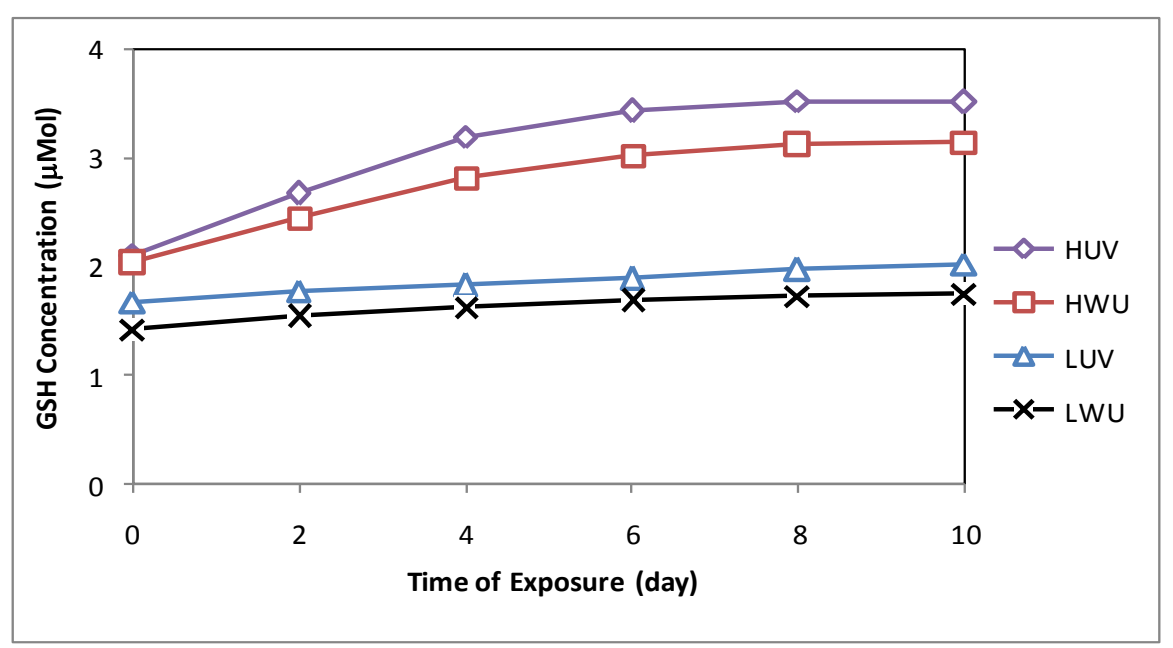

Figure 2. Average concentration of $\mathrm{GSH}(\mu \mathrm{Mol})$ in biomass of Symbiodinium at different time of exposure (day). Treatments: Low radiance with UV (LUV), low radiance without UV (LWU), high radiance with UV (HUV), and high radiance without UV (HWU)

reactions of photorespiratory occur at membrane lipids and proteins, and can further destroy cellular organization (Balarinova et al., 2014). In the course of such destructive reaction the formation of free radicals is augmented in all cellular compartments. Foyer and Noctor (2000) described that described systems may related with a numerous of different functions, such as protection against excess energy absorption, alternative regeneration of the electron acceptor (NADP), the repair of oxidatively damaged structures, the scavenging of ROS, and regulation processes.

In autotrophic cells, the main locations of ROS production are the chloroplasts (Foyer and Noctor, 2000). Furtherly described, that the most common mechanism of stress induced ROS production in the chloroplasts is based on an imbalance between the consumption of reductant (NADPH) in carbon fixation, and the need of the electron transport chain for the regenerated electron acceptor at the PS I site (NADP). Meanwhile the total rates of the mitochondrial ROS production are considered to be much lower than in the chloroplast, the electron transport chains in the mitochondria could be a potential source for side-reactions producing ROS.

\section{Conclusions}

The present work highlighted the role of light on Biomass and GSH production of Symbiodinium cells. The irradiance (include UV exposure) increased stress on Symbiodinium biomass and leaded to induced high intracellular GSH production in Symbiodinium cells. The change of intracellular GSH production indicated the specific photoadaptation mechanism of Symbiodinium.

\section{References}

Ahner, L.P.W., Oleson, J.R. \& Ogura, N. 2002. Glutathione and other low molecular weight thiols in marine phytoplankton under metal stress. Mar. Ecol. Prog. Ser. 232: 93-103.

Al-Farawati, R. \& van den Berg, C.M.G. 2001. Thiols in coastal watres of western North Sea and English Channel. Environ. Sci. Technol. 35: 1902-1911. doi: 10.1021/es000073i

Dupont, C.L., Goepfert, T.J., Lo, P., Wei, L. \& Ahner, B.A. 2004. Diurnal cycling of glutathione in marine phytoplankton: field and culture studies. Limnol. Oceanogr. 49(4): 991-996

Balarinova, K., Bartak, M., Hazdrova, J., Hajek, J. \& Jilkova, J. 2014. Changes in photosynthesis, pigment composition and glutathione contents in two Antartic Lichens during a Light Stress and Recovery. Photosynthetica. 52: 278-288

Finney, J.C., Pettay, D.T., Sampayo, E.M., Warner, M.E., Oxenford, H.A. \& LaJeunesse, T.C.. 2010. The relative significance of host habitat, depth, and geography on the ecology, endemism, and speciation of coral endosymbiont in the genus Symbiodinium. Microb. Ecol. 60: 250-263. doi: 10.1007/s00 248-010-9681-y

Foyer, C.H. \& Noctor, G. 2000. Oxygen processing in photosynthesis: a molecular approach. New Phytol. 146: 359-388. doi: 10.1007/s00248010-9681-y

Krueger, T. \& Gates, R.T. 2012. Cultivating endosymbionts-host environmental mimic support the survival of Symbiodinium C15 ex 
Hospite. J. Exp. Mar. Biol. Ecol. 413: 169-176. doi: 10.1016/j.jembe.2011.12.002

Latowski, D., Goss, R., Bojko, M. \& Strzalkar, K. 2012. Violaxanthin and diadinoxanthin deepoxidation in various model lipid systems. Acta Biochimica Polonica. 59(1): 101-103.

Merwe, R.V., Rothig, T., Voolstra, C.R., Ochsenkuhn M.A., Lattemann, S. \& Amy, G.A. 2014. High salinity tolerance of the Red Sea Coral Fungia granulosa under desalination concentrate discharge conditions: an in situ photophysiology experiment. Front. Mar. Sci. 1: 1-8. doi: 10.3389/fmars.2014.00058

Moya, A., Tambutte, S., Tambutte, E., Zoccola, D., Caminiti, N., \& Allemand, D. 2006. The study of calcification durring daily cycle of the coral Stylophora pistilata: implications for light enhanced calcification. J. Exp. Biol. 209: 34133419. doi: 10.1242/jeb.02382

Muhaemin, M. 2008. The glutathione diurnal cycling in Dunaliella salina. J. Coast. Dev. 12(1): 41-46.

Muhaemin, M. 2011. Dynamic response of ultra violet absorbing in Dunaliella sp. Maspari J.. 3: 20-23.

Osinga, R., Janssen, M. \& Janse, M. 2008. The role of light in coral physiology and its implications for coral husbandry. Advance in Coral Husbandry in Public Aquariums. 2: 173-183.

Perez, S.F., Cook, C.B. \& Brooks, W.R. 2001. The role of symbiotic dinoflagellates in the temperature induced bleaching response of the subtropical Sea Anemone Aiptasia pallida. J. Exp. Mar. Biol. Ecol. 256: 1-14.

Pinto, E., Teresa, C.S.S., Maria., A.S.L., Oswaldo, K.O., Morse, D. \& Colepicolo, P. 2003. Heavy metal induced oxidative stress in algae. J. Phicol. 39: 1008-1018.

Somathilake, L.W., \& Wedagedera, J.R. 2012. On the stability of a mathematical model for coral growth in a tank. British J. Math. Comp Sci. 2(4): 255-280. doi: 10.9734/BJMCS/2012/1 387

Tang, D., Hung, C.C., Warnken, K.W. \& Santschi. P.H. 2000. The distribution of biogenic thiols in surface waters of Galveston Bay. Limnol. Oceanogr. 45: 1289-1297.

Tiltyanov, E.A., Tiltyanov, T.V., Yamazato, K. \& Van Woesik, R. 2001. Photoacclimation dynamics of the coral Stylophora pistillata to low and extremely low light. J. Exp. Biol. Ecol. 263: 211 225. doi: 10.1016/S0022-0981(01)00309-4. 